\title{
A Service-Value Approach to Mobile Application Valuation
}

\author{
Maurizio Cavallari ${ }^{1}$, Roberto Moro Visconti ${ }^{1}$ \\ ${ }^{1}$ Department of Business Administration, Università Cattolica del Sacro Cuore, \\ Largo Gemelli 1 - 20123 Milano, Italy \\ \{maurizio.cavallari@unicatt.it, roberto.morovisconti@morovisconti.it\}
}

\begin{abstract}
Mobile Application Software (M-Apps) are increasingly popular and by now represent the interactive trendiest software. Investigations about their valuation paradigms are so increasingly common. Even if M-Apps belong to the broad category of Intellectual Property assets, their underlying business model is so innovative and different from traditional intangibles that they require new valuation paradigms. The main research question of this paper is to investigate about Service as a primary value driver of M-Apps. A Service-Value-Approach is proposed as a new appraisal method, which embodies customers' perception of M-Apps service value. The empirical evidence fully confirms the hypothesis of the mediating role of Service Quality on application value. This study has practical implications for both scholars and professionals as it provides significant empirical evidence of the role of Service Quality into M-Apps valuation, and value co-creation between providers and users.
\end{abstract}

Keywords: M-Apps; valuation; technology value; service quality; application value.

\section{Introduction}

M-Apps, (a shortening of the term "Mobile Application Software") represent a computer program (software) designed to run on mobile devices such as smart-phones, tablet computers, phablets, smart watches or other mobiles, such as notebooks (with specific extensions).

M-Apps are increasingly popular and by now represent the trendiest software device. Investigations about their valuation paradigms are so increasingly common. Even if M-Apps belong to the broad category of Intellectual Property (IP) assets, their underlying business model is so innovative and different from traditional intangibles (such as patents, brands, etc.) that standard appraisal patterns, normally used for IP, may only be used as a starting point for valuation.

The research question of this paper is to investigate about Service Quality as the main value driver propositions in banking/payment M-Apps valuation, to introduce an innovative valuation approach. The paper is organized as follows: after an introduction about the different M-Apps typologies, traditional IP valuation methods are proposed and challenged. A Service Value Approach (SVA) is then theoretically presented as an innovative valuation method. An empirical investigation is then conducted considering key parameters such as technological value and service quality. The proposed valuation model embeds literature gaps and trendy scenarios, even considering Internet of Things. Network theory, linking developers with users, is also briefly considered, leaving room for further research avenues. Hypothesis about Service value significance into banking/payment M-Apps are developed and tested. An empirical investigation is then pursued to verify the SVA model and determinants. 